\title{
Happy birthday to us
}

\author{
Nature Plants has now completed a full year of publication as a journal aimed at all the plant sciences. \\ What better time to assess the extent to which this goal has been met?
}

Please forgive our self-indulgence in using this Editorial to look back on our first year of publication. This initial period is very important for any journal in showing what it hopes to achieve. Of course, it will be years before an objective assessment of success can be made, but what we have published in 2015 does give a fair impression of the kind of journal we are and will be, both in terms of the breadth of topics covered and the range of content we have produced.

From a research standpoint, every month has had its highlights. We kicked off in January with an issue that included a study by Rute R. da Fonseca et al., who used DNA analysis of archaeological samples to track the routes by which maize cultivation moved out of Mexico and into the Southern United States. It is still one of our most read papers, and produced the memorable News and Views title "Corn in the USA" (sorry, Mr Springsteen).

February's crop of papers contained an ethnobotanical study from Cassandra Quave and Andrea Pieroni looking at the uses of native flora by populations living in the Balkans. By observing how two different populations live in the same area and yet are effectively isolated by their ethnicity, it could be determined whether particular uses of plants were maintained by cultural history or by utility.

We have also been publishing molecular-scale studies. For example, March saw the publication of a paper from Daniel Szymanski and colleagues, who used a combination of live-cell imaging and finite element computational modelling to investigate how actin networks interact with the cell wall to create the characteristic shapes of trichomes. Later on, and also concerning the cytoskeleton, Jonsson et al. and Walter et al. showed how kinesin-14 molecular motors can act in concert to produce smooth, retrograde motion along microtubules to fulfil a similar role to that of dynein in animal cells.

There have also been genetic and molecular biology studies, such as the demonstration by Jerzy Paszkowski and colleagues that heterosis could be seen when crossing individuals who are genetically the same but epigenetically different. Another example is the study by Endang Septiningsih and colleagues, which showed that the trehalose-6-phosphate phosphatase gene OsTPP7 is involved in enabling rice varieties to germinate in anaerobic (that is, submerged) conditions.

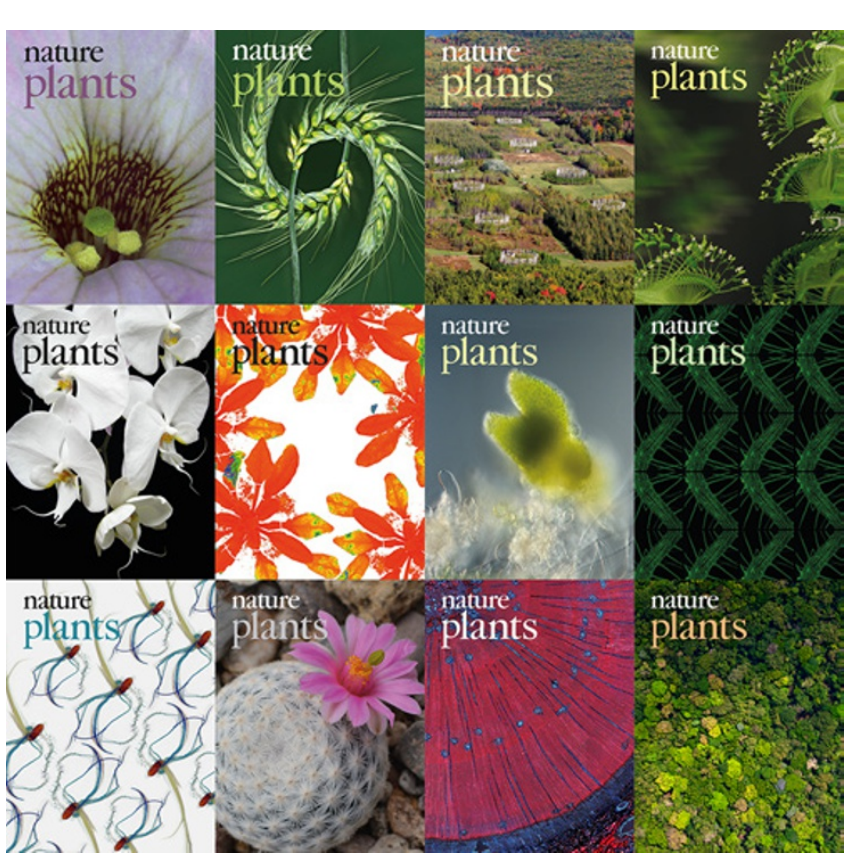

Throughout the year, we have published investigations into the details of photosynthesis, such as Mathew Johnson and colleagues carefully dissecting the part played by the antenna system of light harvesting complex I in the state transitions that adjust the flow of electrons through the photosynthetic machinery to best suit the light conditions. But we have also published studies taking a more global view - such as the analysis by Kristina Anderson-Teixeira and co-workers of worldwide tree growth and mortality data showing that larger trees are more susceptible to drought - as well as conservation studies, including an alarming analysis of cacti by Bárbara Goettsch et al., who concluded that a little more than $30 \%$ of cactus species are threatened with extinction.
With publications from such a wide range of research areas, we hope that we have demonstrated how serious we are about covering the entirety of plant science.

However, we have done more than publish primary research papers. There have been News and Views articles giving scientists opportunities to comment on new works published both in Nature Plants and elsewhere. A prime example would be Jonathan Jones's discussion of a study in the Proceedings of the National Academy of Sciences USA that identifies an Agrobacterium-derived T-DNA insertion as the founding mutation of sweet potato; a 'natural GM' crop, if you will.

Our comment section has been a platform for the discussion of issues relating to modern agriculture and the need to sustainably increase yields. We have also celebrated the 150th anniversary of the publication of Julius Sachs' monograph Handbuch der Experimental-physiologie der Pflanzen (which can be credited with founding the field of plant physiology) and learned that Isaac Newton had his own theories about how water got to the tops of trees.

There isn't space here to list every article we have published over the past year - and we haven't even mentioned the Reviews and News Features. That may be no bad thing, as Nature Plants is a forward-looking journal and, while we are proud of our first year, we are already concentrating on what will fill our second and subsequent volumes. We will continue the breadth of coverage of the plant sciences that we have established in 2015 and expand it further. We will continue to seek out the highest quality and most influential research. And we will continue to strive to be the journal that gives the study of plants the prominence it deserves.

But before putting 2015 behind us completely, we should take a moment to celebrate the beauty that is inherent in plant biology - exemplified by the twelve cover images of our monthly issues. They are a better illustration of the diversity of Nature Plants than any number of words. 